\title{
Beneficial and ineffective levels of selenium for growth and muscular dystrophy
}

\author{
By I. D. DESAI \\ Division of Human Nutrition, School of Home Economics, \\ The University of British Columbia, Vancouver, Canada
}

(Received 28 December 1967-Accepted 21 May 1968)

\begin{abstract}
I. The effectiveness of supplementing a vitamin E-deficient basal diet with levels from o to $20 \mathrm{ppm}$ of selenium as sodium selenite, alone and in combination with graded levels of L-cystine, sodium sulphate, L-serine and vitamin E, has been studied in chicks from I day of age to 4 weeks. Supplementation with Se at levels up to $1 \mathrm{ppm}$ had maximum beneficial effects on growth and prevention of muscular dystrophy. Levels of Se above $1 \mathrm{ppm}$ were progressively less effective, and, in chicks given supplements of $20 \mathrm{ppm}$ Se, growth and muscular dystrophy were depressed to that found with chicks given the basal diet.

2. Supplementation with L-cystine $(0.08 \%$ and $0.16 \%)$ in combination with Se up to I ppm was beneficial, but this effect was progressively suppressed as the amount of Se was increased beyond this level. Supplementation with sodium sulphate $(0.08 \%$ and $0.16 \%)$ or L-serine $(0.08 \%$ and $0.16 \%)$ instead of L-cystine had no influence on the effectiveness of Se at any level of the latter tested.

3. The effectiveness of dietary $D-\alpha$-tocopheryl acetate, up to $20 \mathrm{mg} / \mathrm{kg}$, for growth and in the prevention of muscular dystrophy was improved by Se at levels up to I ppm. Its effectiveness was then progressively reduced as the amount of Se was increased above I ppm.

4. It appears that Se at low levels spares L-cystine and vitamin $\mathbf{E}$ in the chick, whereas at high levels it increases their requirement for growth and prevention of muscular dystrophy.
\end{abstract}

Selenium in trace amounts is recognized as being essential for chicks and perhaps other species, whereas in excessive amounts it is toxic. A clear-cut distinction between the essential and the toxic levels of Se for a number of biological functions is not yet available. It plays an important part, however, not only in maintaining growth but also in preventing a wide variety of deficiency diseases such as liver necrosis in rats (Schwarz \& Foltz, I957), exudative diathesis in chicks (Nesheim \& Scott, I958), and nutritional muscular dystrophy in lambs (Muth, Oldfield, Remmert \& Schubert, 1958) and chicks (Dam \& Søndergaard, 1957; Nesheim \& Scott, 1958).

It has been observed that Se is effective in reducing the muscular dystrophy of chicks, but the degree of its effectiveness was found to be dependent on the presence of a supplemental amount of L-cystine or vitamin E (Scott, 1962). This indication of an interaction between Se, L-cystine and vitamin $\mathrm{E}$ initiated the present investigation. A study is reported on growth and muscular dystrophy of chicks in response to various low and high levels of dietary Se, in the form of sodium selenite, fed singly and in combination with L-cystine and $D$ - $\alpha$-tocopheryl acetate. An attempt is made to differentiate between the beneficial levels and the ineffective levels of Se for growth and for the prevention of nutritional muscular dystrophy in chicks. 
EXPERIMENTAL

One-day-old White Plymouth Rock $\times$ Vantress male chicks, obtained from hens maintained for several generations on a vitamin E-low diet (Nesheim, 1959), were used in this study. Chicks in lots of ten were housed together in electrically heated brooders and were given feed and water ad lib. The control vitamin E-deficient diet was similar to a casein-isolated soya protein-torula yeast diet as described by Scott \& Calvert (I962) with modifications as follows: the $N, N$ 'diphenyl-p-phenylenediamine and sodium selenite were omitted and the diet was supplemented with $0.0125 \%$ I,2-dihydro-6-ethoxy-2,2,4-trimethylquinoline (ethoxyquin) to prevent encephalomalacia and with $\mathrm{I} \cdot 0 \% \mathrm{~L}$-arginine hydrochloride. This diet was fed alone and in combination with graded levels of selenite-selenium (0-20 ppm), L-cystine $(0.08 \%$ and $0.16 \%)$, sodium sulphate $(0.08 \%$ and $0.16 \%)$, L-serine $(0.08 \%$ and $0 \cdot 16 \%)$ and $D-\alpha$-tocopheryl acetate $(0-20 \mathrm{mg} / \mathrm{kg}$ diet). At the end of the 4-week experimental period all chicks were killed and scored for muscular dystrophy. Individual chicks were examined for muscular dystrophy by a panel of three judges scoring independently and without any prior knowledge of the treatments. The scoring was based on an arbitrary scale ranging from zero for absence of dystrophy to 4 for maximal dystrophy. The dystrophic symptoms consisted of distinct necrotic white striations located bilaterally on pectoral muscles. The severity of the disease was determined by the percentage of the total muscle area covered by such striations.

\section{RESULTS}

Fig. I shows the effect of supplementing the basal diet with o-20 ppm Se. Below I ppm, Se had a beneficial effect on growth and on the prevention of muscular dys-

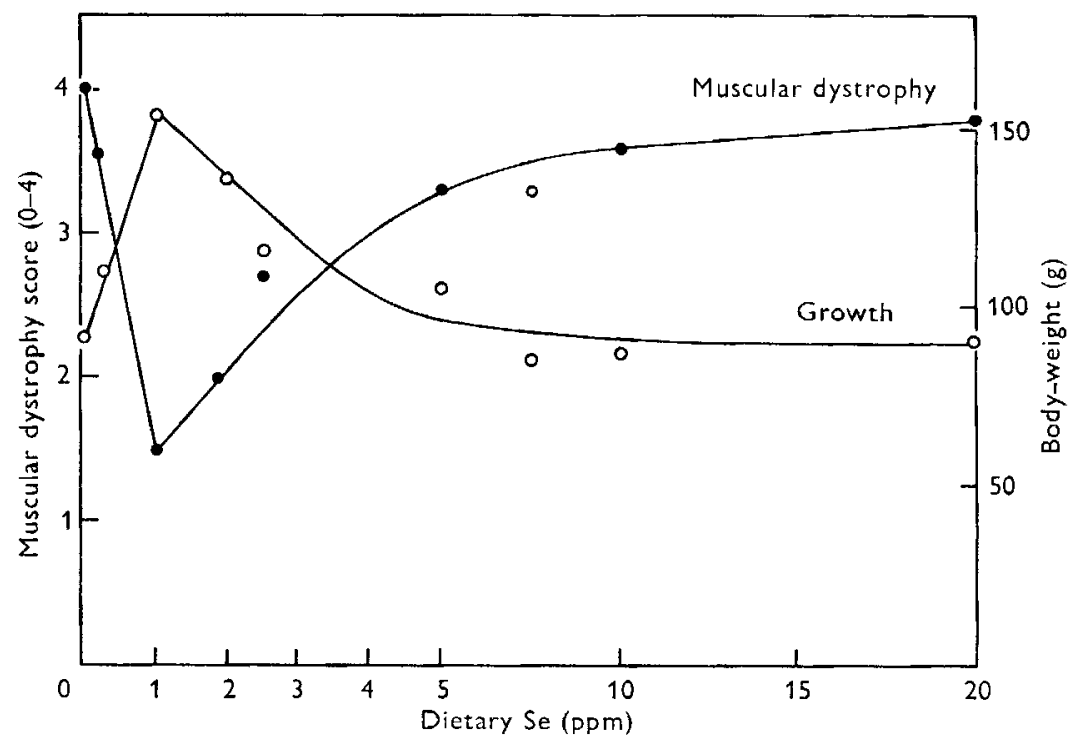

Fig. I. Growth and muscular dystrophy score of chicks given a vitamin E-deficient diet with various low and high levels of selenium. 
trophy. Above I ppm, its effectiveness was progressively reduced until, at $20 \mathrm{ppm}$, it was completely ineffective and growth and muscular dystrophy were similar to those found with the Se-deficient diet.

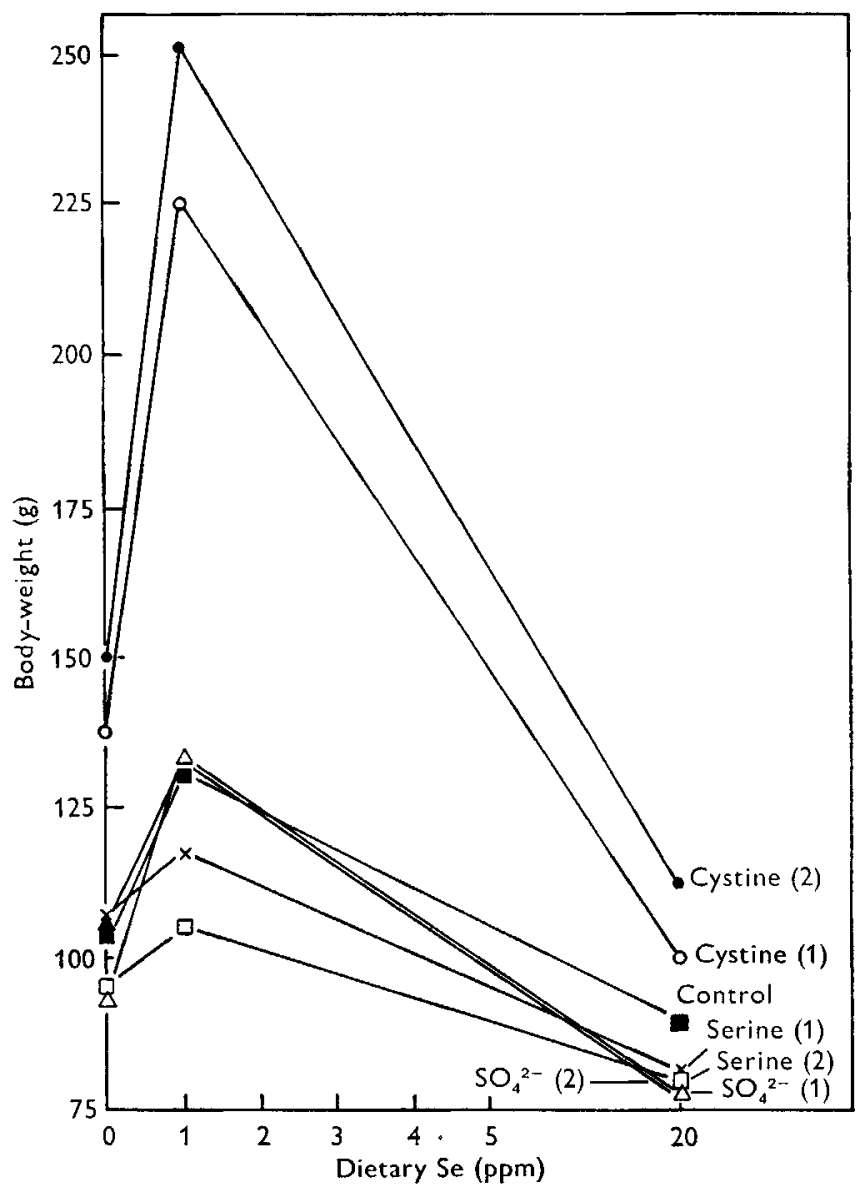

Fig. 2. Growth of chicks given a vitamin E-deficient diet with various levels of selenium alone and in combination with L-cystine, inorganic sulphate and L-serine. (I) represents $0.08 \%$ level and (2) represents $0.16 \%$ level.

Results showing the effectiveness of Se in the presence and absence of L-cystine and equimolar levels of inorganic sulphate and L-serine on growth and muscular dystrophy are presented in Figs. 2 and 3 respectively. Supplementation with L-cystine in combination with I ppm Se was apparently beneficial for growth and prevention of muscular dystrophy, but this effect was progressively reduced as the amount of Se was increased above I ppm. Addition of inorganic sulphate and L-serine instead of L-cystine was ineffective or even decreased the effectiveness of Se with respect to both growth and muscular dystrophy.

Figs. 4 and 5 show that the effectiveness of $D$ - $\alpha$-tocopheryl acetate for growth and 
in the prevention of muscular dystrophy was improved by Se but only up to I ppm of the latter. Beyond this level of Se the effectiveness of $D-\alpha$-tocopheryl acetate progressively decreased.

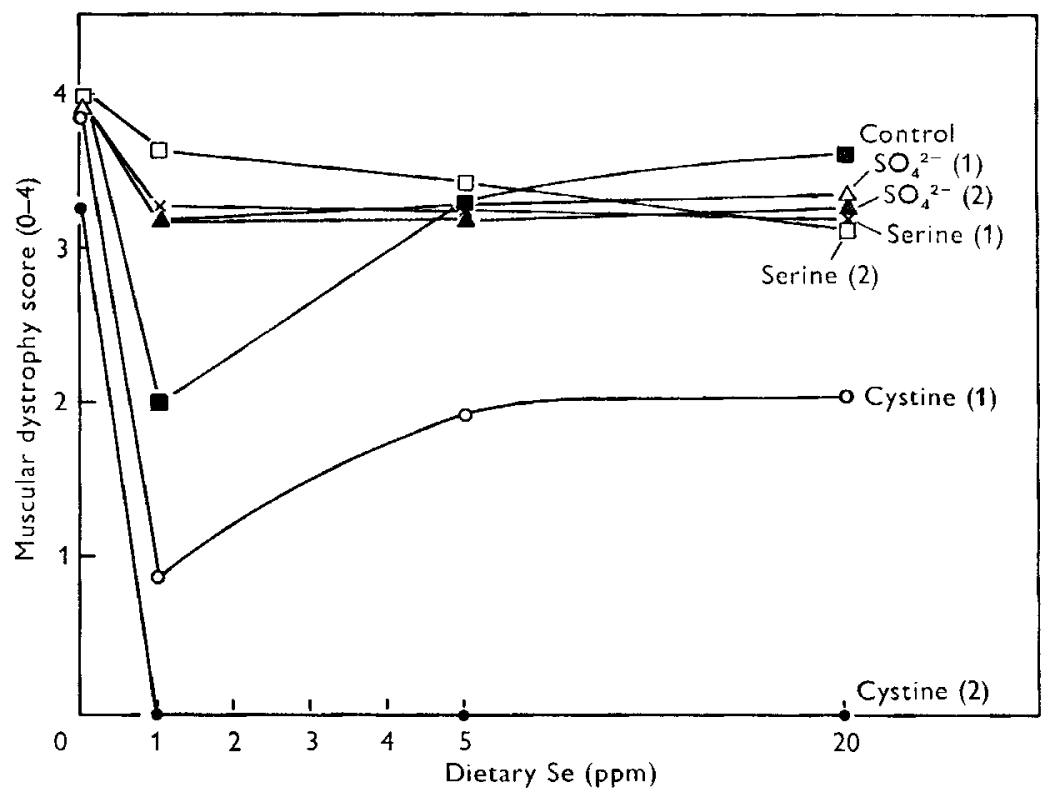

Fig. 3. Muscular dystrophy score of chicks given a vitamin E-deficient diet with various levels of selenium alone and in combination with L-cystine, inorganic sulphate and L-serine. (I) represents $0.08 \%$ level and (2) represents $0.16 \%$ level.

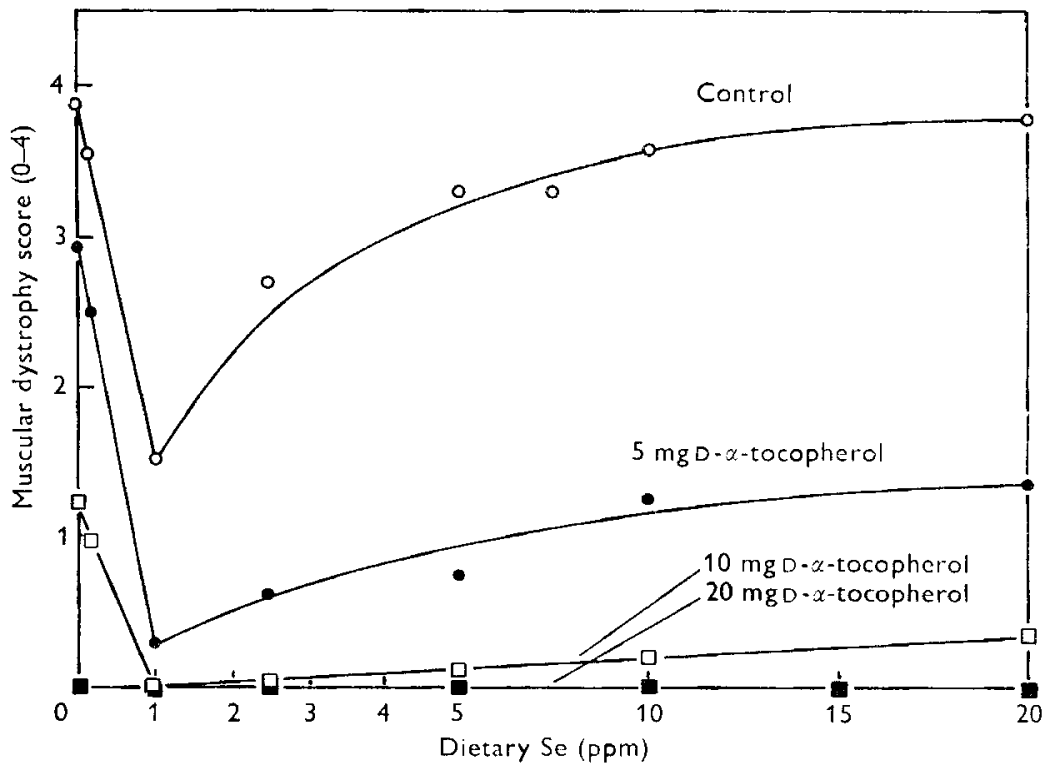

Fig. 4. Muscular dystrophy score of chicks given a vitamin E-deficient diet with various levels of selenium alone and in combination with supplements of $\mathrm{D}$ - $\alpha$-tocopheryl acetate. 


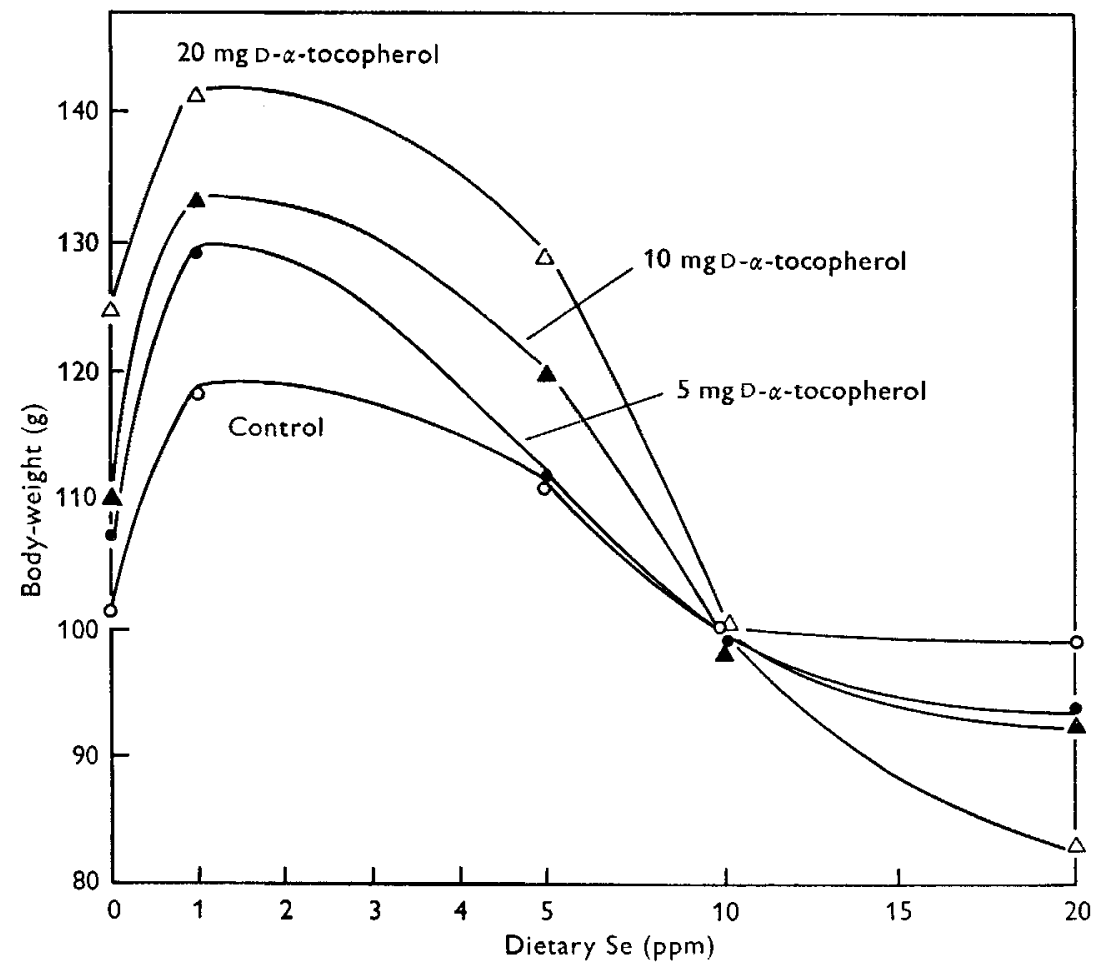

Fig. 5. Growth of chicks given a vitamin E-deficient diet with various levels of selenium alone and in combination with supplements of $D-\alpha$-tocopheryl acetate.

\section{DISCUSSION}

Early studies by Nesheim \& Scott (1958) suggested that the addition of Se as sodium selenite somewhat reduces the incidence of muscular dystrophy in chicks receiving a vitamin E-deficient diet low in methionine and cystine. However, the studies presented in this paper show that the effectiveness of Se is dose-dependent and is related to the presence of L-cystine and vitamin $\mathrm{E}$. The observed effects of Se were of three kinds, depending on its dietary level: (I) beneficial, (2) ineffective, and (3) detrimental or toxic. The beneficial effect for growth and prevention of nutritional muscular dystrophy was observed only with Se levels below I ppm; above this level the effectiveness of Se was progressively reduced until it was completely ineffective or even detrimental.

In a study of the role of Se in growth and for the prevention of muscular dystrophy, it was observed that the growth-depressing effect of high levels of Se could not be reversed by cystine or inorganic sulphate (Fig. 2). Studies on rats (Halverson, Guss \& Olson, 1962) also showed that Se toxicity could not be reversed by the addition of sodium sulphate to diets containing more than ro ppm of Se.

In view of the differences in the effectiveness of high and low levels of Se for growth and muscular dystrophy, it appears that Se may have more than one role, depending on the level at which it is administered. At low levels it seems partially to spare the 
requirement for L-cystine and vitamin $\mathrm{E}$, whereas at higher levels it seems to suppress the effectiveness of $\mathrm{L}$-cystine and vitamin $\mathrm{E}$, thereby increasing their requirement.

It is possible that some Se from the dietary selenite becomes incorporated into cystine to form selenocystine and subsequently selenoproteins, which may be the biologically active forms of Se. The ineffectiveness of high levels of Se seems related to the reduction of selenite to metallic Se, as is observed in Se toxicity. Excessive accumulation of metallic Se perhaps then interferes with the utilization of L-cystine and vitamin $\mathrm{E}$ for growth and prevention of muscular dystrophy.

Using ${ }^{75} \mathrm{Se}$ and ${ }^{3} \mathrm{H}$-tocopherols, Desai \& Scott (1965) have shown that the mode of action of Se is related to the formation of selenolipoproteins, which effectively bind the serum tocopherols. This hypothesis is supported by the finding (Lewis, Quaife \& Page, I954) that in human subjects $74 \%$ of the serum vitamin $\mathrm{E}$ is bound and carried by the serum lipoproteins. The exact biochemical nature of such biologically active complexes involving Se and vitamin $\mathrm{E}$ interactions remains to be investigated.

The author wishes to thank Dr M. L. Scott of Cornell University for his interest, encouragement and expert advice.

\section{REFEREN CES}

Dam, H. \& Søndergaard, E. (1957). Experientia I3, 494.

Desai, I. D. \& Scott, M. L. (1965). Archs Biochem. Biophys. 110, 309.

Halverson, A. W., Guss, P. L. \& Olson, O. I. (1962). F. Nutr. 77, 459.

Lewis, L. A., Quaife, M. L. \& Page, I. H. (I954). Am. F. Physiol. 178, 221.

Muth, O. H., Oldfield, J. E., Remmert, L. F. \& Schubert, J. R. (1958). Science, N.Y. r28, 1090.

Nesheim, M. C. (r959). PhD Thesis, Cornell University.

Nesheim, M. C. \& Scott, M. L. (I958). F. Nutr. 65, 6ox.

Schwarz, K. \& Foltz, C. M. (1957). F. Am. chem. Soc. 79, 3292.

Scott, M. L. (1962). Nutr, Abstr. Rev. 32, 1.

Scott, M. L. \& Calvert, C. C. (1962). F. Nutr. 77, 105. 